\title{
Histological study of follicular atresia in the ovary of the domestic hen (Gallus domesticus)
}

\author{
S. K. Gupta*, A. B. Gilbert and M. A. Walker \\ AFRC Institute of Animal Physiology and Genetics Research, Edinburgh Research Station, Roslin, \\ Midlothian EH25 9PS, U.K.
}

\begin{abstract}
Summary. Semi-serial ( 1 in 20 ) sections of ovaries were studied and only two types of atresia were identified-non-bursting and bursting. Smaller, non-yolky follicles ( $<1 \mathrm{~mm}$ diameter) showed non-bursting atresia. Atresia in follicles $>1 \mathrm{~mm}$ diameter was invariably of the bursting type which involved the rupture of the follicular wall, and the extrusion of yolk and cellular debris through the rupture site into the stroma. However, this rupture site was small and consequently was not visible in every section but it could always be seen when the follicle was followed in semi-serial sections. The mitotic index of granulosa cells in bursting atretic follicles was much lower than that for normal follicles.

The most common criteria for distinguishing non-bursting atretic follicles were the extremely shrunken, irregularly shaped oocytes and the separation of the granulosa from the theca. In bursting atretic follicles, reliable indications were the presence in the ooplasm of some cells or cellular debris, and disorganization of the yolk and granulosa tissue. The presence of pycnotic nuclei in the granulosa cells was not a consistent feature of all atretic follicles of the hen.
\end{abstract}

Keywords: hen; follicles; atresia; histology

\section{Introduction}

Unlike mammals (Byskov, 1978; Ryan, 1981; Guraya, 1985), histological criteria for the identification of atretic follicles in the avian ovary are not well established. This has led to subjective assessment of atresia in birds, and the use of different names and systems of classification; e.g. 2-6 types of follicular atresia in birds have been reported (see Gupta \& Maiti, 1986). It remains doubtful whether these discrepancies are real or are the result of problems related to technique and/or interpretation (Gilbert, 1979; Gupta, 1983). Confusingly, similar names appear to have been used for dissimilar atretic structures and vice versa. In their reviews, Saidapur (1978) and Gilbert (1979) categorized all the atretic structures within the avian ovary which were described in earlier papers into two basic types, i.e. bursting and involution atresia. Bursting atresia, involving rupture of the follicular wall with extrusion of ooplasmic material, was claimed to be the least common type. Involution (or invasion) atresia has been reported to resemble atresia in the mammalian ovary and involved reabsorption of the atretic mass in situ. This has been thought to be the most common type. However, Gupta \& Maiti (1986) have demonstrated that, in the ovary of the Indian pied myna, all larger follicles ( $>500 \mu \mathrm{m}$ diameter) undergo bursting atresia whereas atresia of smaller follicles $(<500 \mu \mathrm{m}$ diameter) is of the non-bursting type. Whether the atretic process is similar in the ovary of the domestic hen remains to be determined because evidence from earlier studies is equivocal (Fell, 1925; Deol, 1955; Uhrin, 1984).

The present investigation in the hen's ovary was carried out with two objectives: (1) to provide histological criteria for the identification of atretic follicles, and (2) to provide a simpler integrated view regarding the types of atresia present.

*Present address: Department of Zoology, M.L.N. College, Yamuna Nagar, Haryana, India-135001. 


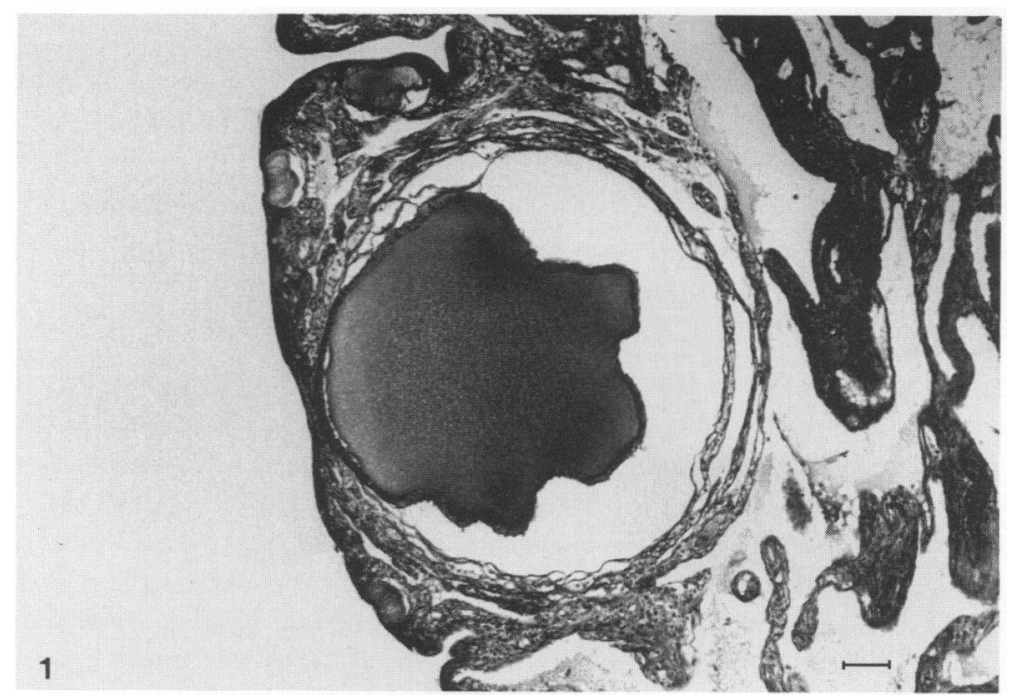

Fig. 1. An atretic follicle of non-bursting type. Note the shrunken oocyte, and the granulosa separated from the theca. Bar $=100 \mu \mathrm{m}$.

\section{Materials and Methods}

Eight laying hens of domestic fowl (Gallus domesticus) were housed in individual cages with food and water available ad libitum, and they were provided with $14 \mathrm{~h}$ light $(04: 30-18: 30 \mathrm{~h}) / 24 \mathrm{~h}$ day. They were killed by an intravenous injection of an overdose of pentobarbitone sodium (Expiral: Ceva Laboratories, Southampton, U.K.). Ovaries were quickly removed and follicles $>8 \mathrm{~mm}$ diameter were discarded since the incidence of atresia in these is rare (Gilbert $e t$ al., 1983). Smaller follicles projecting from the ovarian surface and small pieces of the body of the ovary were taken from the anterior and posterior regions since evidence is available that the ovary may be divided into two functional regions (anterior and posterior) demarcated by a narrow band (D. Waddington, personal communication). The tissues were fixed in Bouin's or Susa fluid and were processed routinely. Semi-serial sections ( 1 in every 20 ) were cut at $5 \mu \mathrm{m}$ and stained by Mallory's or Masson's trichrome technique.

For the study of mitotic activity in the granulosa cells, 4 birds were housed similarly but with lights on from 12:00 $\mathrm{h}$ to $02: 00 \mathrm{~h}$ for 10 days for acclimatization. Colchicine is known to arrest cell division at the metaphase stage and it (Sigma Chemical Co., Poole, Dorset, U.K.) was administered intraperitoneally at $0 \cdot 1 \mathrm{mg} / \mathrm{kg}$ body weight at 10:00 $\mathrm{h}$. In a preliminary study evidence was obtained that mitotic cells were more frequently seen at this time. The birds were killed $4 \mathrm{~h}$ later and tissue sections were prepared as described above. The numbers of metaphase cells in 10 normal and 10 atretic follicles per ovary ( 3 sections per follicle) were counted. For each section, the total numbers of metaphase cells present in the granulosa layer were counted; use of a grid or frame was found unsuitable for the purpose of counting these cells because of shrinkage of the atretic follicles.

\section{Results}

Atretic follicles were a common feature in all the ovaries studied. On first examination, various different types of atretic structures were thought to be present but closer examination of semi-serial sections revealed that these atretic structures could, in fact, be placed in only two clearly defined categories, i.e. Type 1 "non-bursting atresia" and Type 2 "bursting atresia".

\section{Non-bursting atresia (Type 1)}

This type of atresia was characteristically found in small follicles, $<1 \mathrm{~mm}$ diameter. It was characterized by shrinkage of the oocyte which assumed an irregular shape (Fig. 1). In addition, the granulosa was separated from the theca by a wide empty space and was irregularly folded. Balbiani's vitelline body was indistinct. Both the oocyte and the granulosa had altered staining 


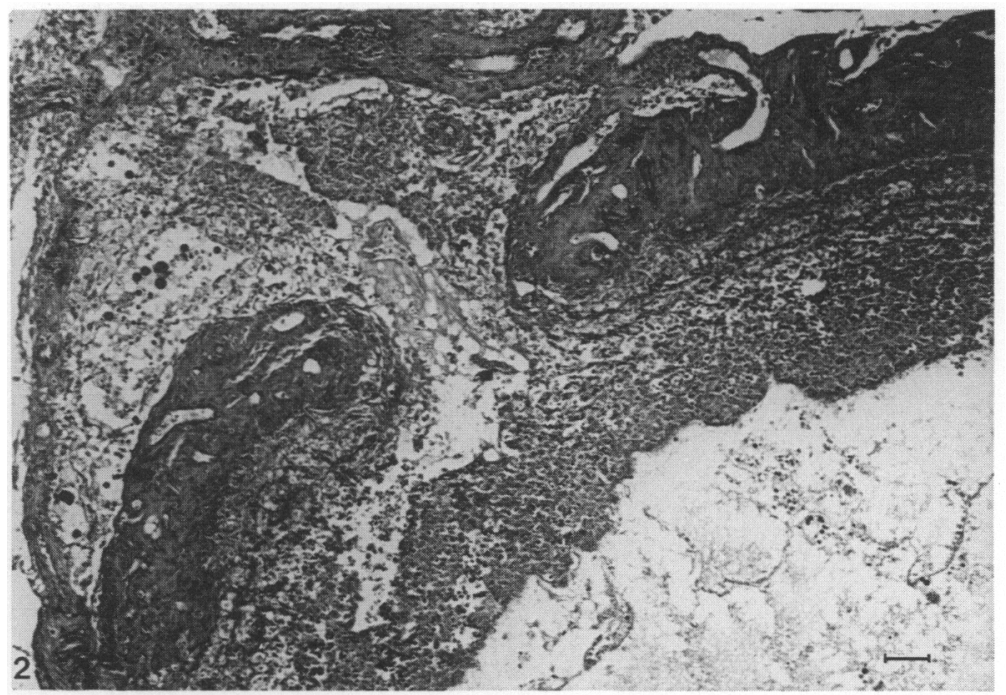

Fig. 2. The rupture site of a bursting type of atretic follicle. Ooplasmic contents escape through the rupture site and are seen to lie outside the follicle. Bar $=100 \mu \mathrm{m}$.

properties in that they were stained more darkly and diffusely. The thecal elements appeared loose and there was no hypertrophy or increased collagenization. The follicular wall was not ruptured.

\section{Bursting atresia (Type 2)}

Atresia in follicles larger than $1 \mathrm{~mm}$ diameter was typically of the bursting type. This was characterized by the rupture of the follicular wall, extrusion of the ooplasmic contents through the rupture site into the ovarian stroma and the presence of several cell types (e.g. granulosa, thecal, blood) in the ooplasm (Figs 2,3). The rupture occurred usually through a single and small breach, which could be located at any point of the follicle with the exception of the stigma region. There was usually hypertrophy and increased collagenization of the theca. However, in some follicles, the granulosa cells appeared to pass out through the thecal layer at several sites. In contrast to the usual appearance, there was neither hypertrophy nor collagenization of the theca in these cases (Fig. 4). Typically, in all cases, the oocyte nucleus was degenerate. A marked disorganization of the yolk substances, the perivitelline layer, the granulosa and the theca interna (Table 1) was also observed. The rupture site was discernible even in the last stages of atresia and at all stages the stigma-like area was present. These last two features allowed an atretic follicle to be distinguished from the post-ovulatory follicle in the final stages of regression.

A summary of the major histological criteria of atresia in the hen's ovary are given in Table 1 and the major differences between Type 1 and Type 2 atresia are summarized in Table 2 . Not all of these histological features were observed in each particular section and nor were they observed in every atretic follicle, but they were common to follicles in the anterior and posterior regions of the ovary. Moreover, it is not possible to indicate any single criterion which would be consistently seen in every atretic follicle.

Treatment with colchicine resulted in cell divisions being arrested at the metaphase stage, thus enabling an estimate to be made of the relative rates of cell divisions in the granulosa tissue from normal and atretic follicles. There were fewer dividing cells in the granulosa from Type 2 atretic follicles $(9.8 \pm 2.0$ per section) than in the comparable normal follicles $1-8 \mathrm{~mm}$ in diameter $(62.4 \pm 6.5$ per section). These 'metaphase cells' were also observed in normal follicles smaller than $1 \mathrm{~mm}$ diameter but they were not clearly identifiable in comparable Type 1 atretic follicles. 


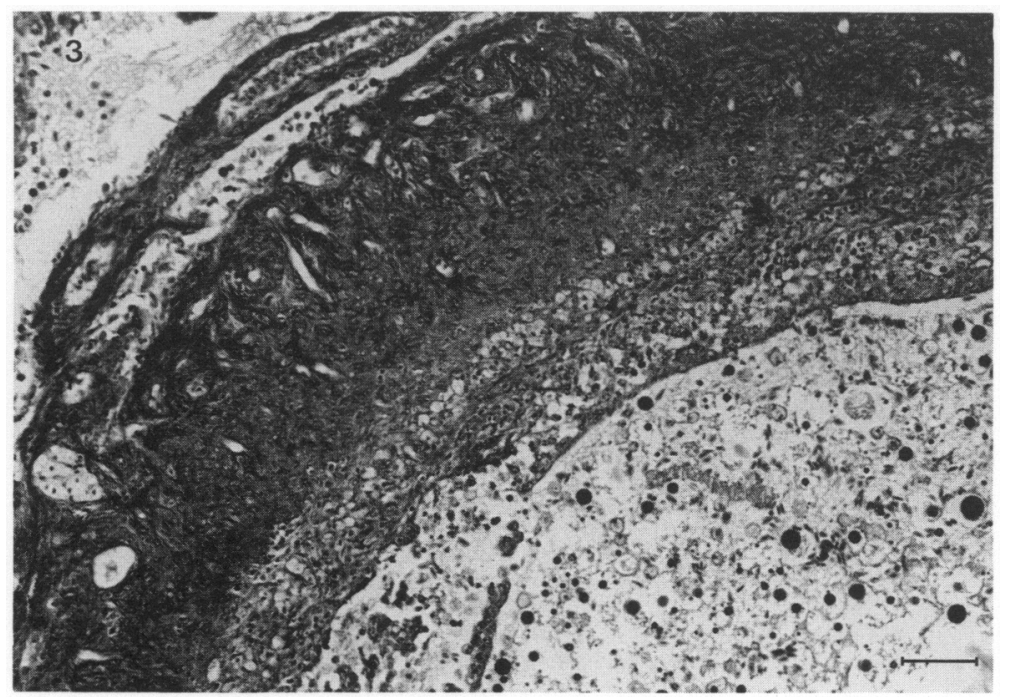

Fig. 3. Part of a bursting atretic follicle showing disorganized granulosa and yolk, and the presence of various cells in the ooplasm. Thecal hypertrophy, vacuolization in theca interna cells, collagenization of theca externa, disrupted basal lamina and perivitelline layer (zona pellucida) are evident. Bar $=100 \mu \mathrm{m}$.

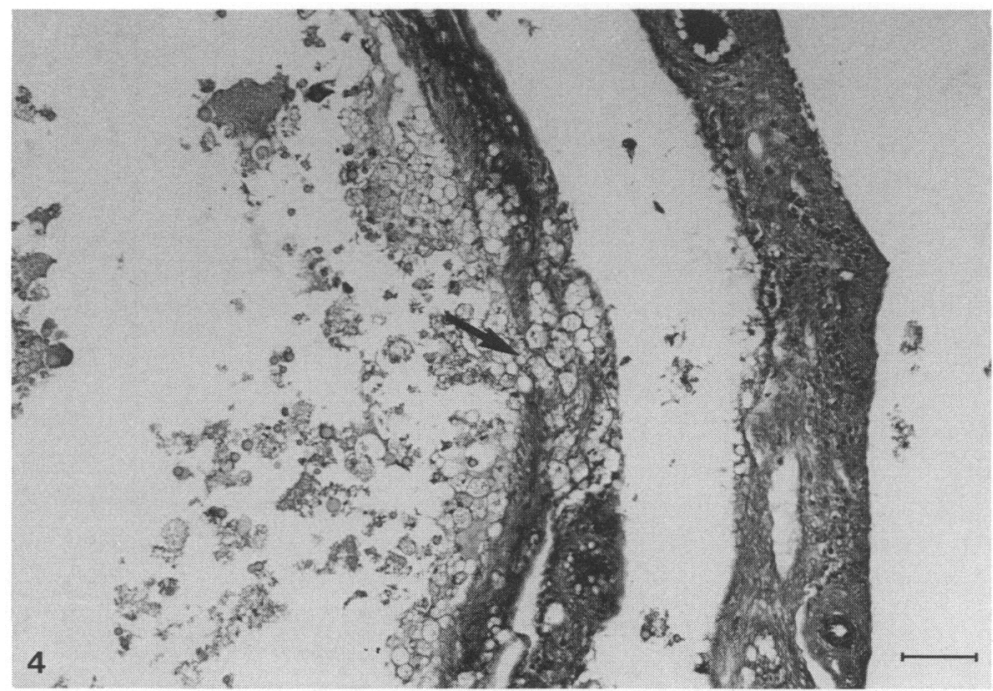

Fig. 4. A less commonly observed type of bursting atretic follicle. In semi-serial sections of such follicles, granulosa cells were found to pass out through the theca at several sites (arrow). Bar $=100 \mu \mathrm{m}$. 
Table 1. Histological criteria of atresia (Types 1 and 2) in the domestic hen's ovary

\begin{tabular}{ll}
\hline Oocyte & (1) Shrunken irregularly shaped oocyte \\
& (2) Disorganisation of yolk \\
& (3) Presence of ex-granulosa cells, ex-thecal cells, blood cells or cellular debris in the ooplasm \\
Perivitelline & (1) Shrinkage, hyalinization and fragmentation of the perivitelline layer \\
layer & (2) Separation of the perivitelline layer from the granulosa \\
Granulosa & (3) Wavy appearance \\
& (1) Dissociation among granulosa cells \\
& (3) Decreased mitotic activity \\
Basal lamina & (4) Increased height or multi-layered appearance \\
Theca interna & (1) Fragmentation \\
& (1) Increased height, vacuolations and extracellular spaces; increased numbers of 'clear' \\
Theca externa & (2) Invasion of granulosa; loss of identity of granulosa and theca interna \\
& (1) Increased collagenization \\
& (3) Haemorrhage; prominent radial blood channels across theca \\
Miscellaneous & (1) Rupture of follicle wall \\
& (2) Yolk and cellular debris outside the follicle \\
& (3) Reduction in follicle size
\end{tabular}

Table 2. Important differences between non-bursting and bursting types of atresia in the domestic hen's ovary

\begin{tabular}{ll}
\hline Non-bursting atresia & Bursting atresia \\
\hline No rupture of follicular wall & Involves rupture of follicular wall \\
Occurs in follicles $<1 \mathrm{~mm}$ in diameter & Occurs in follicles $>1 \mathrm{~mm}$ in diameter \\
Reabsorption of atretic mass in situ & $\begin{array}{l}\text { Ooplasmic contents along with cellular debris escape } \\
\text { through the rupture site into the ovarian stroma; } \\
\text { reabsorption mainly outside the follicle }\end{array}$ \\
$\begin{array}{l}\text { Characterized by the presence in the ooplasm of ex- } \\
\text { granulosa, ex-theca or blood cells, and disorganisation } \\
\text { of yolk and granulosa }\end{array}$ \\
\hline \begin{tabular}{l} 
empty space \\
\hline
\end{tabular}
\end{tabular}

\section{Discussion}

Our findings on follicular atresia in the ovary of the domestic hen are not in agreement with those reported earlier (Fell, 1925; Deol, 1955; Uhrin, 1984). By using semi-serial sections of the ovary in the present study it was possible to show that in the hen there are only two types of atresia present which is in contrast to the several types reported previously (Gilbert, 1979). These two types are related to the developmental stage or size of the follicle that becomes atretic. The smaller follicles, $<1 \mathrm{~mm}$ diameter, undergo non-bursting atresia (Type 1) whereas atresia in all follicles larger than $1 \mathrm{~mm}$ in diameter is invariably of the bursting type (Type 2). By considering the three dimensional picture of the follicle with a small rupture site on one side, it is obvious that the probability is large of missing this point of rupture in histological sections. Possibly this is the most likely explanation 
why earlier workers, who examined only a few representative sections of the ovary, did not appreciate such frequent occurrence of bursting atresia in the hen (Fell, 1925; Deol, 1955; Uhrin, 1984) or most other birds (see references in Gupta \& Maiti, 1986). Different kinds of atretic structures considered earlier to be the involution or invasion type (Saidapur, 1978; Gilbert, 1979) were in fact always found in semi-serial sections to be of Type 2 . What causes rupture of the follicular wall remains unknown. However, although described as such only occasionally, the occurrence of Type 2 atretic follicles has been recorded in many avian species (Davis, 1944; Gilbert, 1979). Moreover, in more mature follicles, which were not considered in the present investigation, atresia has been reported to be of Type 2 (Davis, 1942; Yoshimura \& Tamura, 1985). The atretic process in birds is therefore different from that in mammals and is apparently related to the large amounts of yolk in the later stages of oocyte maturation in birds.

The present study failed to identify the site where the atretic changes first appear. The present evidence, though inconclusive, favours the oocyte itself since in all cases the oocyte nucleus had already degenerated by the time other aspects of atresia developed. Further investigations are required to identify the precise mechanisms responsible for initiating the process which culminates in atresia.

Since, previously, bursting of atretic follicles was observed only rarely, it is widely believed that reabsorption of yolk occurs in situ, i.e. within the follicle. Our results show that this is not usually the case because in Type 2 atretic follicles, which are common, the yolk escapes through the rupture site into the stroma and hence is outside the follicle. A similar observation was made by Gupta \& Maiti (1986) in the Indian pied myna ovary.

Another widely held assumption is that the increased height of the granulosa layer at the beginning of atresia is due to an increased cell proliferation. However, no specific tests for studying mitotic activity were carried out in previous studies. By using colchicine-injected hens it was possible to show that mitotic activity in the granulosa cells of atretic follicles was less than that in the granulosa of normal follicles. Similar observations have been reported for mammals (Ryan, 1981). This is consistent with the view that follicular atresia is a degenerative rather than a growth process. The apparently increased granulosa height may merely reflect the decrease in the diameter of the atretic follicle through loss of yolk.

In contrast to mammals (Byskov, 1978; Ryan, 1981; Guraya, 1985), the appearance of pycnotic nuclei in the granulosa layer of the hen's ovary was not the most reliable histological indication of atresia, although they were encountered. Instead, the most common features of atretic follicles were the shrunken irregularly shaped oocyte with the granulosa separated from the theca in Type 1, and the presence of several cell types in the ooplasm with disorganized yolk and granulosa in Type 2 .

We have also observed that normal (healthy) follicles may sometimes be mistaken for atretic ones under the microscope depending upon the plane of the section or the area of the follicle at which the section was cut. For example, a section passing through one side of a normal follicle would appear to be smaller in size than a normal section with an indistinct perivitelline layer and basal lamina, an apparent increased granulosa cell number or height and an apparent hypertrophied theca features usually associated with an atretic follicle. Similarly, if the section passed through the theca alone, it would appear as if the follicle consisted only of a group of hypertrophied gland cells together with connective tissue cells. Such structures have indeed been reported to be the last stages of atresia in which the ooplasm and the invading granulosa cells have been completely reabsorbed leaving a group of thecal gland cells in the stroma. However, these errors of interpretation are avoided if serial or semi-serial sections are used in which the true nature of the follicle becomes evident.

It has been repeatedly reported that it is not possible to distinguish between the late stages of regression of a post-ovulatory follicle and the late stages of atresia (Gilbert, 1979; Gupta, 1983, for references). This is incorrect because in the hen two features of an atretic follicle make such a distinction possible, i.e. (1) the site of rupture in the ovarian stroma and (2) the stigma-like area, neither of which are present in the post-ovulatory follicle. 
S.K.G. thanks The Rotary Foundation of Rotary International, U.S.A., for scholarship grants and the Principal and Managing Committee of M.L.N. College, Yamuna Nagar, India, for encouragement.

\section{References}

Byskov, A.G. (1978) Follicular atresia. In The Vertebrate Ovary, pp. 533-562. Ed. R. E. Jones. Plenum Press, New York.

Davis, D.E. (1942) Bursting of avian follicles at the beginning of atresia. Anat. Rec. 82, 153-161.

Davis, D.E. (1944) The occurrence of burst atretic follicles in birds. Anat. Rec. 90, 307-309.

Deol, G.S. (1955) Studies on the structure and function of the ovary of the domestic fowl (with special reference to the correlation of cell changes with physiological activity). Ph.D thesis, University of Edinburgh.

Fell, H.B. (1925) Histological studies on the gonads of the fowl. III. The relationship of the 'luteal' cells of the ovary of the fowl to the tissue occupying the atretic and discharged follicles, and the question of the homology of the latter tissue and the mammalian corpus luteum. Q. Jl microsc. Sci. 69, 591-609.

Gilbert, A.B. (1979) Female genital organs. In Form and Function in Birds, Vol. 1, pp. 237-360. Eds A. S. King \& J. McLelland. Academic Press, London.

Gilbert, A.B., Perry, M.M., Waddington, D. \& Hardie, M.A. (1983) Role of atresia in establishing the follicular hierarchy in the ovary of the domestic hen (Gallus domesticus). J. Reprod. Fert. 69, 221-227.
Gupta, S.K. (1983) Reproductive biology of the Indian pied myna, Sturnus contra contra (L.) Ph.D thesis, University of Calcutta.

Gupta, S.K. \& Maiti, B.R. (1986) Study of atresia in the ovary during the annual reproductive cycle and nesting cycle of the pied myna. J. Morph. 190, 285-296.

Guraya, S.S. (1985) Biology of Ovarian Follicles in Mammals. Springer-Verlag, Berlin.

Ryan, R.J. (1981) Follicular atresia: some speculations of biochemical markers and mechanisms. In Dynamics of Ovarian Function, pp. 1-11. Eds N. B. Schwartz \& M. Hunzicker-Dunn. Raven Press, New York.

Saidapur, S.K. (1978) Follicular atresia in the ovaries of non-mammalian vertebrates. Int. Rev. Cytol. 54, 225-241.

Uhrin, V. (1984) Follicle atresia in the growing ovary of fowl. Vet. Med., Praha 29, 181-188.

Yoshimura, Y. \& Tamura, T. (1985) Histological and histochemical observations on the atretic follicles induced by adenohypophysectomy in the hen. Jpn. Poult. Sci. 22, 134-141.

Received 29 April 1987 